\title{
Highlights of 33rd EANM Annual Congress 2020, virtual edition
}

\author{
Giovanni Matassa $^{1,2} \cdot$ Fabrizia Gelardi $^{1,2} \cdot$ Roland Hustinx $^{3} \cdot$ Arturo Chiti $^{1,2}$ \\ Published online: 28 April 2021 \\ (C) The Author(s), under exclusive licence to Springer-Verlag GmbH Germany, part of Springer Nature 2021
}

\section{Introduction}

The 33rd Annual Congress of the European Association of Nuclear Medicine (EANM) took place from 22 to 30 October 2020 in an unusual virtual edition due to the SARSCoV-2 outbreak under Professor's Fanti chairmanship. Nevertheless, scientific content level and attendance remained high, confirming the EANM congress's record as the most important event of the year in the nuclear medicine community not only in Europe but worldwide. The virtual edition allowed 3034 participants to join the congress from anywhere globally (more than 110 countries), reaching 170,446 hits on the virtual platform. All European countries attended with great enthusiasm, but many international colleagues from the USA, Japan and Australia actively contributed to the congress (Fig. 1).

All major topics of interest in the nuclear medicine community were discussed in more than 100 sessions of high scientific standards. Every session was characterised by a multidisciplinary involvement of all professional figures that revolve around the world of nuclear medicine, including radiopharmacists and chemists, physicists, technologists, physicians and researchers. Overall, among the 1540 submitted abstracts, 1311 (1243 scientific, 68 technologists) were accepted, while the number of rejected was 229. EU countries, including Italy (12\%), Spain (11\%) and Germany (7\%),

Giovanni Matassa and Fabrizia Gelardi contributed equally to this work.

This article is part of the Topical Collection on Miscellanea

\section{Arturo Chiti}

arturo.chiti@hunimed.eu

1 Department of Biomedical Sciences, Humanitas University, Via Rita Levi Montalcini 4, Pieve Emanuele, 20090 Milan, Italy

2 IRCCS Humanitas Research Hospital, Via Manzoni 56, Rozzano, 20089 Milan, Italy

3 Division of Nuclear Medicine and Oncological Imaging, University Hospital of Liège, and GIGA-CRC in vivo imaging, University of Liège, Liège, Belgium
France $(6 \%)$ and the UK (5\%), submitted the greatest number of abstracts. The non-EU country that has most contributed was China (5\%) (Fig. 1). During 163 sessions, physicians and technologists exposed 846 oral presentations and 397 e-posters, leading to exciting debates.

This paper aims to give an overview of the works presented in the highlights lecture of the EANM 2020 congress.

\section{Real-world data}

Nowadays, nuclear medicine has a remarkable place in the clinical setting owing to its major impact in several healthcare fields. It provides notable diagnostic and therapeutic contributions leading to patients' well-being and quality of life improvement.

In this scenario, PSMA ligand imaging has latterly received increased attention, playing a pivotal role in prostate cancer management, including initial staging, biochemical recurrence detection, treatment planning and response evaluation. Afshar-Oromieh et al. [1, 2] investigated the performance of $\left[{ }^{68} \mathrm{Ga}\right] \mathrm{Ga}-\mathrm{PSMA}-11 \mathrm{PET} / \mathrm{CT}$ in a large cohort of patients with recurrent prostate cancer $(\mathrm{PCa})$. Patients with untreated primary $\mathrm{PCa}$, undergoing androgen-deprivation therapy and those previously treated with chemotherapy or exclusively external beam radiation therapy, were excluded. They found a clear association between tumour detection and higher PSA levels and a higher Gleason Score. No association was found with injected activity or clinical parameters such as age, PSA doubling-time or PSA velocity. Rodnick et al. [1] evaluated prospectively the main failure patterns based on initial treatment of PCa performing $\left[{ }^{68} \mathrm{Ga}\right] \mathrm{Ga}-\mathrm{PSMA}-11$ PET/CT. They observed a higher rate of local recurrence after radiotherapy and higher rates of nodal and osseous metastases after radical prostatectomy. A prospective randomised phase 3 clinical trial was carried out by Calais et al. [1] They focused on the success rate of salvage radiotherapy (SRT) for recurrent PCa after prostatectomy with versus without planning based on $\left[{ }^{68} \mathrm{Ga}\right] \mathrm{Ga}$-PSMA-11 PET. Preliminary results showed that 
EANM 2020 submitted abstracts

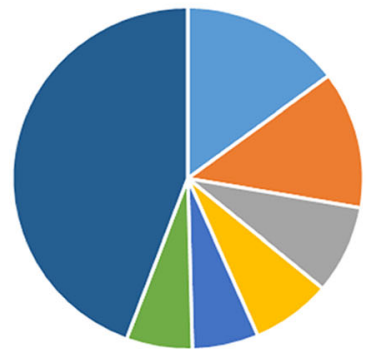

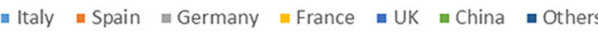

EANM 2020 Participants

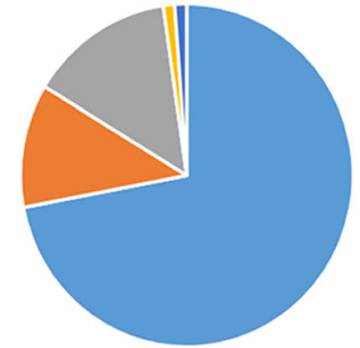

Europe $\|$ America $\|$ Asia - Oceania $\|$ Africa

Fig. 1 Abstracts submitted to 33rd EANM Annual Congress 2020 by country (on the left). Italy, Spain, Germany, France and the UK submitted the greatest number of abstracts. China was a non-EU country that participated more actively. Participants to the congress by continent (on the right)

PET impacted the RT plan in more than half of the patients determining a change or abortion of the SRT plan.

Besides its diagnostic use, the PSMA ligand imaging is increasingly performed before and during PSMA-based radio-ligand therapy (RLT). Indeed, ${ }^{177}$ Lu-labelled PSMA ligands have recently been demonstrated to be a safe and effective therapeutic strategy for the treatment of mCRPC. Patientspecific dosimetry is expected to enhance the safety of molecular radiotherapy treatments and to predict response. MRTDosimetry was a European project with the main goal of standardising quantitative Lu-177 SPECT/CT imaging. Via multicentre intercomparison of eight SPECT/CT systems, Tran-Gia et al. [1] proved that reliable quantitative SPECT/ $\mathrm{CT}$ is feasible when following the calibration protocol developed within MRTDosimetry.

Concerning radionuclide therapy, Lambert et al. [1] compared the efficacy of $\mathrm{Y}-90$ radioembolisation (TARE) with drug-eluting beads chemoembolisation (DEB-TACE) for treating hepatocellular carcinoma (HCC) in patients not eligible for surgery or thermo-ablation. They assessed the time to progression (TTP) at 2 years according to modified Response Evaluation Criteria In Solid Tumors (mRECIST), time to local tumour progression (TLP) in the treated liver volume at 2 years, safety (adverse events and 30-day mortality) and overall survival. This randomised controlled trial revealed that TARE was superior to DEB-TACE for tumour control and survival, whereas safety was similar for both groups.

In paediatric oncology, Fayolle et al. [1] studied the prognostic value of the metabolic tumour volume, $\mathrm{SUV}_{\max }$, $\mathrm{SUV}_{\text {peak }}$ or bone lysis measured from a pre-treatment $\left[{ }^{18} \mathrm{~F}\right]$ FDG PET/CT scan in childhood rhabdomyosarcoma (RMS), taking into account overall survival and progression-free survival. The results underlined that a metabolic tumour volume greater than $200 \mathrm{~cm}^{3}$ was an independent predictor of poor outcome.
Quantitative accuracy of PET imaging in longitudinal clinical assessments is a challenge when data are acquired using different scanners and protocols. Huff et al. [1] described a retrospective harmonisation process to solve this issue. The proposed method for quantitative harmonisation used deconvolution and optimal filtering and reduced differences caused by imaging hardware and reconstruction settings for a retrospective PET dataset.

Another interesting work was presented by Caobelli et al. [1]. They carried out a 5-year follow-up of the prospective multicentre trial BARDOT. The trial had already demonstrated that asymptomatic diabetic patients with abnormal myocardial perfusion scintigraphy (MPS) are at increased risk of major cardiac events (MACE) at a 2-year follow-up. Abnormal MPS was defined as the summed stress score (SSS) $\geq 4$ or summed difference score (SDS) $\geq 2$. MACEs were defined as myocardial infarction, cardiac death or late coronary revascularisation. The present study confirmed that if patients with diabetes are clinically at high risk of CAD as in BARDOT, they should be considered for ischemia testing. Conversely, a routine scan predicts a good 5-year outcome.

\section{Towards the clinic}

Several novel radiopharmaceuticals have been synthesised and introduced in various clinical settings to overcome some of the limitations and challenges currently faced by the tracers used in daily practice. Among them, radiolabeled fibroblastactivation-protein inhibitors (FAPIs) seem particularly promising. Staudinger et al. [1] investigated their in vivo biodistribution in 80 patients with head and neck, lung, pancreatic or colorectal cancer. FAPI radiopharmaceuticals showed a high tumour-to-background ratio thanks to a rapid renal clearance and a reduced extraction in non-target organs, 
as well as a comparable uptake between the primitive lesion and distant metastases.

The L-glutamate derivative $\left[{ }^{18} \mathrm{~F}\right] \mathrm{FSPG}$ is a novel PET tracer designed to study tumour-specific oxidative stress processes through system $\mathrm{x}_{\mathrm{C}}{ }^{-}$activity. Iagaru et al. [1] evaluated $\left[{ }^{18} \mathrm{~F}\right] \mathrm{FSPG}$ potential in patients referred to rule out sarcoidosis and with $\left[{ }^{18} \mathrm{~F}\right] \mathrm{FDG}$ studies negative, equivocal or non-diagnostic. $\left[{ }^{18} \mathrm{~F}\right] \mathrm{FSPG}$ PET/CT showed very encouraging results, with the major advantage that it does not need any dietary restriction prior to imaging, in contrast with $\left[{ }^{18} \mathrm{~F}\right] \mathrm{FDG}$.

In a phase II clinical trial, Paquette et al. [1] assessed the performance of the ER-binding PET radiotracer 4-fluoro-11 $\beta$ methoxy-16 $\alpha-\left[{ }^{18} \mathrm{~F}\right]$ fluoroestradiol (4FMFES) in 20 uterine cancer patients for staging purpose. Compared to $\left[{ }^{18} \mathrm{~F}\right] \mathrm{FDG}$, 4FMFES showed a higher tumour-to-background ratio (17.8 \pm 6.4 vs $7.6 \pm 5.0$ ), while $\left[{ }^{18} \mathrm{~F}\right] \mathrm{FDG}$ better correlated with tumour grade and lesion sizes.

A promising alternative to $\left[{ }^{68} \mathrm{Ga}\right]$-DOTA-peptides is $\left[{ }^{18} \mathrm{~F}\right]$ AlF-NOTA-octreotide: A head-to-head comparison with $\left[{ }^{68} \mathrm{Ga}\right] \mathrm{Ga}$-DOTATATE PET/CT was recently carried out by Pauwels et al. $[1,3]$ in 6 NET patients. $\left[{ }^{18} \mathrm{~F}\right]$ AlF-NOTAoctreotide revealed a favourable biodistribution, a good correlation between $\mathrm{Ki}$ and uptake, a similar tumour-to-background ratio and a high detection rate, which increased over time until reaching the maximum $\mathrm{SUV}_{\max }$ at $3 \mathrm{~h}$ post-injection.

Great enthusiasm is increasingly permeating the scientific community concerning advances in prostate cancer imaging and therapy. Since PSMA has been identified as an excellent target to develop new radiopharmaceuticals, several molecules have been studied to establish the best biodistribution and logistical characteristics in a theranostic perspective. A phase I open-label trial was carried out by Malaspina et al. [1] to evaluate the kinetics of $\left[{ }^{18} \mathrm{~F}\right] \mathrm{rhPSMA}-7.3$ performing a 45-min dynamic and two static PET/CT acquisitions at 60 and 90 min post-injection, respectively. $\left[{ }^{18} \mathrm{~F}\right] \mathrm{rhPSMA}-7.3$ demonstrated excellent performance in lesion detection and increased pathological uptake over time, achieving the best target-to-background ratio at $60 \mathrm{~min}$ post-injection. $\left[{ }^{18} \mathrm{~F}\right]$ rhPSMA-7.3 is a promising radiopharmaceutical for imaging and therapeutic use in prostate cancer.

Paganelli et al. [1], in a phase 2 study, focused on androgen receptors (AR) aberrations, correlated with the onset of CRPC, and their potential role in early response assessment ${ }^{177}$ Lu-PSMA therapy. After determining the circulating AR copy number on plasma samples with droplet digital polymerase chain reaction (ddPCR), $40 \mathrm{mCRPC}$ patients were treated with $\left[{ }^{177} \mathrm{Lu}\right] \mathrm{Lu}-\mathrm{PSMA}-617$. They found a correlation between AR gene gain and the onset of early progressive disease, suggesting that prostate cancer patients in the early phases of disease would respond better to $\left[{ }^{177} \mathrm{Lu}\right] \mathrm{Lu}-\mathrm{PSMA}-617$.

Rottenburger et al. [1] presented preliminary data of two patients from a phase I trial of a novel cholecystokinin-2 receptor agonist ${ }^{177} \mathrm{Lu}$-DOTA-(DGlu)6-AlaTyr-Gly-Trp-Nleu-
Asp-PheNH2 ([ $\left.\left.{ }^{177} \mathrm{Lu}\right]-\mathrm{PP}-\mathrm{F} 11 \mathrm{~N}\right)$ for radionuclide therapy in advanced medullary thyroid carcinoma (MTC). Three injections of $\left[{ }^{177} \mathrm{Lu}\right]-\mathrm{PP}-\mathrm{F} 11 \mathrm{~N}(6 \mathrm{GBq})$ were safe and reported adverse reactions, i.e. mainly hot flushes, nausea and hyperhidrosis, were mostly grade 2 or lower. From dosimetry data, cumulative administrated activity was $18 \mathrm{GBq}$, and no toxicity in normal organs was observed in follow-up visits. Final published results [4] on six patients confirmed [ $\left.{ }^{177} \mathrm{Lu}\right]-\mathrm{PP}-\mathrm{F} 11 \mathrm{~N}$ favourable biodistribution and specific uptake in MTC lesions. However, further dosimetry and clinical studies should be carried out to establish the maximum tolerated dose for the best possible tumour response.

Research and technological advances have led to major developments in long axial field-of-view (FOV) PET/CT. The advantages may be many, in particular acquiring fast and/or low-dose whole-body images with high sensitivity and spatial resolution and also possibly multiple kinetic modelling. Conti et al. [1] evaluated time and spatial resolution, sensitivity and standard phantom image quality of a 106-cm-long axial FOV prototype using Monte Carlo simulation. Preliminary data confirmed excellent sensitivity, spatial and temporal resolution. Phantom image quality remains high even when reducing the activity or the acquisition time.

\section{Data mining}

Advanced imaging analysis, machine learning and their potential application in daily clinical practice have increasingly become a hot topic and a source of debate in the nuclear medicine community in recent years.

Radiomics, representing the high-throughput extraction of quantitative image features, might improve standard visual image analysis, obtaining further valuable information for diagnostic and prognostic purposes. In this context, Eertink et al. [1] performed radiomics analyses on baseline $\left[{ }^{18} \mathrm{~F}\right]$ FDG PET/CT of 296 newly diagnosed diffuse large B cell lymphoma (DLBCL) to identify patients at risk of relapse up to 2 years after treatment. Quantitative radiomics features showed a high predictive value both alone and combined with well-known clinical prognostic factors (age and WHO performance status), revealing the additional advantage of advanced imaging analysis. In particular, a model associating the MTV, $\mathrm{SUV}_{\text {peak }}$ and maximal distance between the largest lesion and any other (Dmaxbulk) yielded the highest predictive value (CV-AUC of $0.75 \pm 0.07$ ).

Deep Learning algorithms are increasingly investigated in nuclear medicine with many potential clinical applications, including the radiation dosimetry assessment in patients undergoing radionuclide therapy. A deep learning-based method for voxel-wise dosimetry prediction of $\left[{ }^{117} \mathrm{Lu}\right] \mathrm{Lu}$-PSMA therapy in 30 metastatic castration-resistant prostate cancer 
(mCRPC) patients was presented by Xue et al. [1]. Data from pre-therapy $\left[{ }^{68} \mathrm{Ga}\right] \mathrm{Ga}$-PSMA-11 PET/CT and post-therapy $\left[{ }^{117} \mathrm{Lu}\right] \mathrm{Lu}$-PSMA SPECT/CT were included using a dualinput-model in 3D radioligand therapy dose generative adversarial networks (GANs), showing an excellent ability to generate accurate dose maps.

Hagmarker et al. [1] have instead used deep convolutional neural networks (CNNs) to produce synthesised projections (SIPs) for post-therapy dosimetry SPECT imaging with $\left[{ }^{177} \mathrm{Lu}\right]$-DOTATATE to reduce the number of sampled projections. The study carried out on 44 patients affected by advanced neuroendocrine tumours demonstrated that the hybrid method with SIPs does not change the bone marrow absorbed dose with the advantage of reducing the acquisition time, keeping the image quality unchanged.

\section{Innovation}

Nuclear medicine has displayed continuous development and rising clinical impact over the last two decades. It contributes to biomedical research in many areas such as oncology, neurological and cardiovascular science, preventive healthcare and many other aspects of medicine.

Building upon the massive clinical success of PSMA ligands, Malaspina et al. [1] presented a prospective singlecentre trial to validate the PSMA-flare phenomenon in hormone-sensitive prostate cancer (Pca) after short-term androgen-deprivation therapy (ADT). A possible correlation between Pca aggressiveness and $\left[{ }^{18} \mathrm{~F}\right] \mathrm{FDG}$-uptake was also evaluated. These preliminary results confirmed a heterogeneous increase in $\left[{ }^{18} \mathrm{~F}\right]$ PSMA-1007 uptake after ADT, predominant in bone metastases. No clear association was found with glucose metabolism.

Speaking of PSMA ligands, Pawlak et al. [1] focused on the preclinical assessment of new PSMA analogue labelled with ${ }^{47} \mathrm{Sc},\left[{ }^{47} \mathrm{Sc}\right] \mathrm{Sc}-\mathrm{PSMA}-\mathrm{D} 4$ and analysed its chemical and biological properties. This new radiopharmaceutical is attractive as it could be used for therapy and also allow imaging using SPECT .

Mesothelin-targeted thorium-227 conjugate (MSLN-TTC, $\left[{ }^{227} \mathrm{Th}\right]$-anetumab corixetan) is a new targeted alpha therapy currently being tested in phase 1 in patients suffering from mesothelioma and serous ovarian cancer. Hagemann et al. [1] compared intravenous and intraperitoneal administration of MSLN-TTC in a murine model of peritoneal carcinomatosis of mesothelioma. Intraperitoneal administration of MSLNTTC resulted in increased efficacy and decreased myelosuppression.

Alpha-emitters may also be applied to peptide receptor radioligand therapy (PRRT), as Tworowska et al. [1] presented a first-in-human study of AlphaMedix ${ }^{\text {TM }}$ ([ $\left.{ }^{212} \mathrm{~Pb}\right]$-DOTAMTATE), a new somatostatin analogue for targeted alpha-emitter therapy in patients with unresectable or metastatic neuroendocrine tumours overexpressing somatostatin receptors. Early results showed that PRRT with ${ }^{212} \mathrm{~Pb}$ is feasible and led to an objective radiological response in all 16 cases with a notable reduction in tumour burden. The treatment was well tolerated, with only mild adverse events.

The overexpression of androgen receptor (AR) is demonstrated in the majority of glioblastoma multiforme (GBM) specimens; Shamni et al. $[1,5]$ evaluated whether $16 \beta-{ }^{18}$ Ffluoro-5 $\alpha$-dihydrotestosterone ( $\left[{ }^{18} \mathrm{~F}\right]$-FDHT) could detect and quantify AR expression in GBM patients, in order to select candidates who might benefit from AR antagonist therapy. Preliminary results in 12 patients indicate that $\left[{ }^{18} \mathrm{~F}\right]$-FDHT PET/CT might be a helpful tool for selecting those patients, also allowing treatment response evaluation.

Beyond continuous molecular imaging advances in the oncological field, many innovative radiopharmaceuticals are currently under development in several non-oncological conditions.

Neuroscience is another major field of research as illustrated by Hesse et al. [1]. They investigated the availability of $\alpha 4(\alpha 6) \beta 2 *$ nicotinic acetylcholine receptor (nAChR) before and after rewarding food cue visual stimulation in individuals with obesity (OB) and normal-weight controls (NW). They performed PET/MRI with (-)-[ $\left.{ }^{18} \mathrm{~F}\right]$ flubatine twice on separate days (rest and stimulation). Disinhibited eating behaviour was assessed using the German version of the Three-Factor Eating Questionnaire (TFEQ). Compared with NW and lowdisinhibited eaters with $\mathrm{OB}, \alpha 4(\alpha 6) \beta 2 *$-nAChR availability of high-disinhibited eaters with $\mathrm{OB}$ appears to be altered in the thalamus, the mesolimbic system and in the basal forebrain. Such a study may be helpful in elucidating the biological substrates of obesity.

Another fascinating abstract was presented by Lerdsirisuk et al. [1]. They designed and synthesised new compounds for tau imaging and then investigated in vitro and in vivo profiles. The results suggested that the new ${ }^{18} \mathrm{~F}$-labelled tracer $\left[{ }^{18} \mathrm{~F}\right] \mathrm{SNFT}-1$ (THK-5562) is a promising candidate selective for Tau PET imaging in Alzheimer's disease, with less offtarget binding.

Bacterial infections are a severe emerging global healthcare challenge. The siderophore-based iron uptake system differs between bacterial and mammalian cells. Petrik et al. [1, 6] demonstrated that deferoxamine, a siderophore marketed under the brand name Desferal (DFO), can be radiolabeled with gallium-68 without loss of bioactivity. In vitro characterisation was tested in different microbial cultures, whereas in vivo biodistribution was studied in healthy mice. PET scans were performed in suitable animal infection models, confirming the potential of $\left[{ }^{68} \mathrm{Ga}\right] \mathrm{DFO}$ for specifically imaging bacterial infections (Fig. 2). 


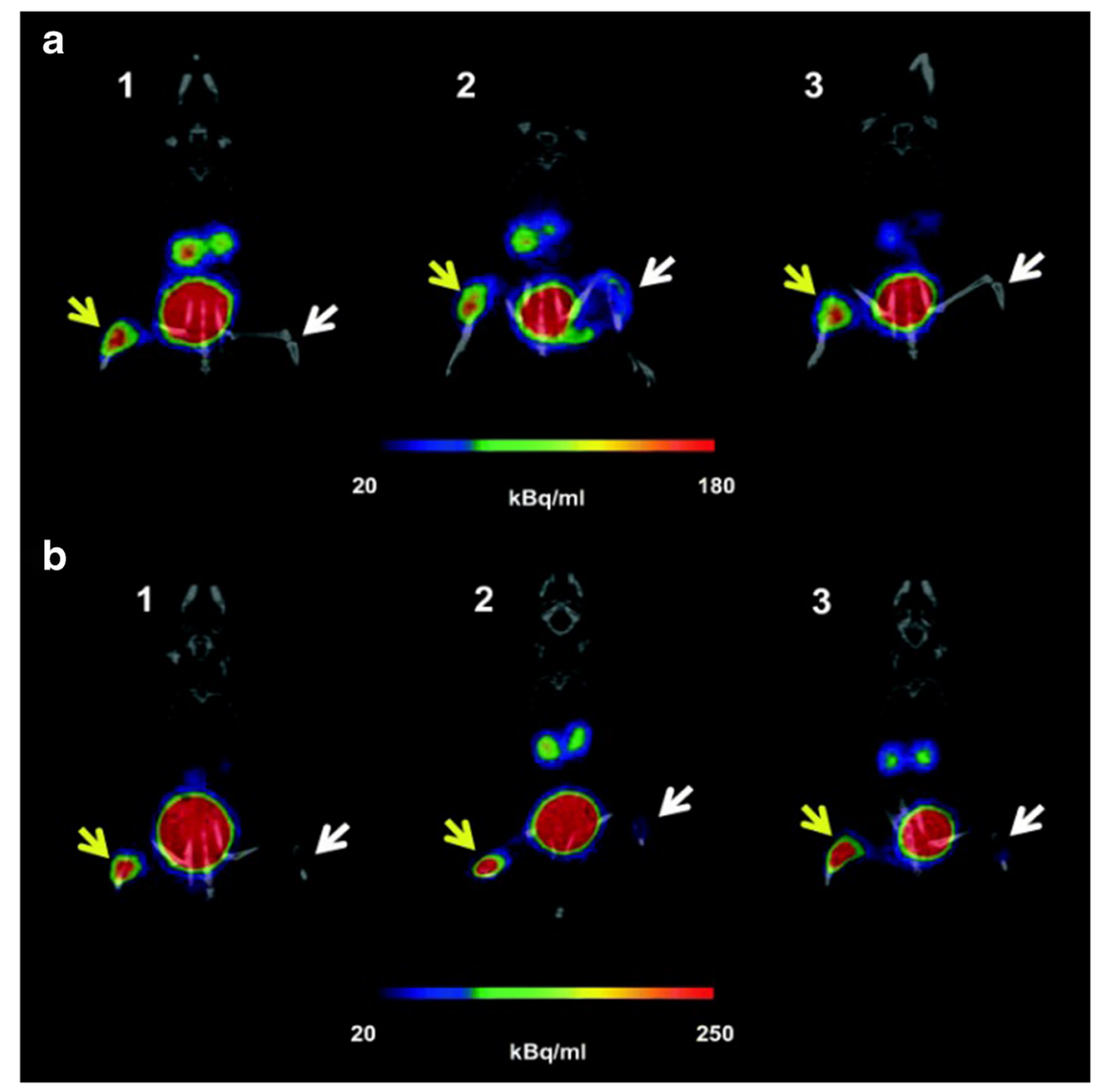

Fig. 2 In vivo uptake of [68Ga]Ga-DFO-B was studied in acute murine myositis models established by intramuscular injection of different bacteria strains (live or heat-killed) or with turpentine oil to induce sterile inflammation. The microbial infections were allowed to develop for $5 \mathrm{~h}$ and sterile inflammation for $24 \mathrm{~h}$. Animals were subsequently injected with [68Ga]Ga-DFO-B and imaged using PET/CT. PET/CT images (coronal slices) of acute murine myositis $5 \mathrm{~h}$ after inoculation and $45 \mathrm{~min}$ after

\section{Conclusions}

As always, the 33rd EANM Annual Congress 2020 confirmed the vibrancy and extraordinary commitment of the nuclear medicine community in research. The scientific contributions presented in these highlights aimed at reflecting the diversity and the vitality of the research programs, covering all medical and scientific fields and revealing some of the most significant trends in the progresses made by nuclear medicine in the diagnostic and therapeutic settings.

\section{Declarations}

Ethical approval Institutional Review Board approval was not required for this paper.

Informed consent Not applicable.

Conflict of interest The authors declare no conflict of interest. injection of [68Ga]Ga-DFO-B (a) $P$. aeruginosa versus $P$. aeruginosa heat-killed (1), $P$. aeruginosa versus sterile inflammation (2), $P$. aeruginosa versus $E$. coli (3); (b) $S$. aureus versus $S$. aureus heatkilled (1), S. aureus versus sterile inflammation (2), S. aureus versus E. coli (3); yellow arrow indicates $P$. aeruginosa or $S$. aureus infection, while a white arrow pointing at $P$. aeruginosa or $S$. aureus heat-killed, sterile inflammation or $E$. coli infection

\section{References}

1. European Journal of Nuclear Medicine and Molecular Imaging. 2020;47(Suppl 1):S1-S753. https://doi.org/10.1007/s00259-020-04988-4.

2. Afshar-Oromieh, A. et al. Performance of [ $68 \mathrm{Ga}$ ]Ga-PSMA-11 $\mathrm{PET} / \mathrm{CT}$ in patients with recurrent prostate cancer after prostatectomy-a multi-centre evaluation of 2533 patients. https:// doi.org/10.1007/s00259-021-05189-3.

3. Pauwels E, et al. [18F]AlF-NOTA-octreotide PET imaging: biodistribution, dosimetry and first comparison with [68Ga]GaDOTATATE in neuroendocrine tumour patients. Eur J Nucl Med Mol Imaging. 2020;47:3033-46.

4. Rottenburger $\mathrm{C}$, et al. Cholecystokinin 2 receptor agonist $177 \mathrm{Lu}-\mathrm{PP}-$ F11N for radionuclide therapy of medullary thyroid carcinoma: results of the Lumed Phase 0a Study. 2020. https://doi.org/10.2967/ jnumed.119.233031.

5. Orevi M, et al. [ 18F]-FDHT PET/CT as a tool for imaging androgen receptor expression in high-grade glioma. Neuro-Oncology Adv. 2021. https://doi.org/10.1093/noajnl/vdab019.

6. Petrik M, et al. 68Ga-labelled desferrioxamine-B for bacterial infection imaging. Eur J Nucl Med Mol Imaging. 2021;48:372-82.

Publisher's note Springer Nature remains neutral with regard to jurisdictional claims in published maps and institutional affiliations. 